\title{
PRESENT STATUS OF IGEX DARK MATTER SEARCH AT CANFRANC UNDERGROUND LABORATORY
}

\author{
I.G. IRASTORZA ${ }^{a * \dagger}$, A. MORALES ${ }^{a}$, C.E. AALSETH ${ }^{b}$, F.T. AVIGNONE III ${ }^{b}$, \\ R.L. BRODZINSKI ${ }^{c}$, J. M. CARMONA ${ }^{a}$, S. CEBRIÁN ${ }^{a}$, E. GARCíA $^{a}$, \\ I.V. KIRPICHNIKOV ${ }^{d}$,A.A. KLIMENKO ${ }^{e}$, G. LUZÓN ${ }^{a}$, H.S. MILEY ${ }^{c}$, \\ J. MORALES ${ }^{a}$, A. ORTIZ DE SOLÓRZANO ${ }^{a}$, S.B. OSETROV ${ }^{e}$, \\ V.S. POGOSOV ${ }^{f}$, J. PUIMEDÓN ${ }^{a}$, J.H. REEVES ${ }^{c}$, \\ M.L. SARSA ${ }^{a}$,A.A. SMOLNIKOV ${ }^{e}$, A.G. TAMANYAN ${ }^{f}$, A.A. VASENKO ${ }^{e}$, \\ S.I. VASILIEV ${ }^{e}$, J.A. VILLAR ${ }^{a}$ \\ The IGEX Collaboration \\ ${ }^{a}$ Laboratory of Nuclear and High Energy Physics, University of Zaragoza, 50009 \\ Zaragoza, Spain \\ ${ }^{b}$ University of South Carolina, Columbia, South Carolina 29208 USA \\ ${ }^{c}$ Pacific Northwest National Laboratory, Richland, Washington 99352 USA \\ ${ }^{d}$ Institute for Theoretical and Experimental Physics, 117259 Moscow, Russia \\ ${ }^{e}$ Institute for Nuclear Research, Baksan Neutrino Observatory, 361609 \\ Neutrino, Russia \\ ${ }^{f}$ Yerevan Physical Institute, 375036 Yerevan, Armenia
}

\begin{abstract}
One IGEX ${ }^{76} \mathrm{Ge}$ double-beta decay detector is currently operating in the Canfranc Underground Laboratory in a search for dark matter WIMPs, through the Ge nuclear recoil produced by the WIMP elastic scattering. In this talk we report on the on-going efforts to understand and eventually reject the background at low energy. These efforts have led to the improvement of the neutron shielding and to partial reduction of the background, but still the remaining events are not totally identified. A tritium contamination or muon-induced neutrons are considered as possible sources, simulations and experimental test being still under progress. According to the success of this study we comment the prospects of the experiment as well as those of its future extension, the GEDEON dark matter experiment.
\end{abstract}

*attending speaker, e-mail: igor.irastorza@cern.ch

${ }^{\dagger}$ Present address: CERN, EP Division, CH-1211 Geneva 23, Switzerland 


\section{Introduction}

Recent cosmological observations and robust theoretical arguments require an important Dark Matter component $\left(\Omega_{D M} \sim 25-30 \%\right)$ in our universe, which is supposed to be made mostly of non-baryonic particles. Weakly Interacting Massive (and neutral) Particles (WIMPs), which are favourite candidates to such non-baryonic component, could fill the galactic halos accounting for the flat rotation curves which are measured for many galaxies. They could be detected by measuring the nuclear recoil produced by their elastic scattering off target nuclei in a suitable detector ${ }^{1}$. The IGEX dark matter experiment is currently operating one germanium detector with this purpose in the Canfranc Underground Laboratory. In this talk we review the present status of the experiment. Following a brief description in section 2, we focus on the on-going work about the identification and rejection of the low energy background in the section 3. In section 4 we will present the prospects of the experiment, as well as of the GEDEON project, a proposed extension of the IGEX dark matter search that will be commented in some detail. We will finish with the conclusions in section 5 .

\section{Experiment}

The IGEX experiment ${ }^{3,4}$, optimized for detecting ${ }^{76} \mathrm{Ge}$ double-beta decay, has been described in detail elsewhere. One of the IGEX detectors of 2.2 $\mathrm{kg}$ (active mass $\sim 2.0 \mathrm{~kg}$ ), enriched up to $86 \%$ in ${ }^{76} \mathrm{Ge}$, is being used to look for WIMPs interacting coherently with the germanium nuclei. The Ge detector and its cryostat were fabricated following state-of-the-art ultralow background techniques and using only selected radiopure material components (see $\operatorname{Ref}^{3,5}$ ).

The detector shielding has been modified several times since the beginning of the dark matter phase of IGEX. The improvements concern basically the neutron moderator, which thickness went from the $20 \mathrm{cms}$ of polyethylene for the first published data ${ }^{5}$ to $40 \mathrm{cms}$ (of polyethylene and borated water tanks) in ${ }^{6}$ and to $80 \mathrm{cms}$ in the more recent data taken. After the first changes of the shielding, it much better covers the whole set-up, due to the removal of the other detectors (RG-I, RG-III and COSME), which dewars did not allow us to perfectly close the polyethylene wall. These changes were motivated by a previous study of the possible sources of background in IGEX based on preliminary simulations, which pointed out that the neutrons from the surrounding rock could contribute considerably to the low energy background. This background study is still going on as commented 


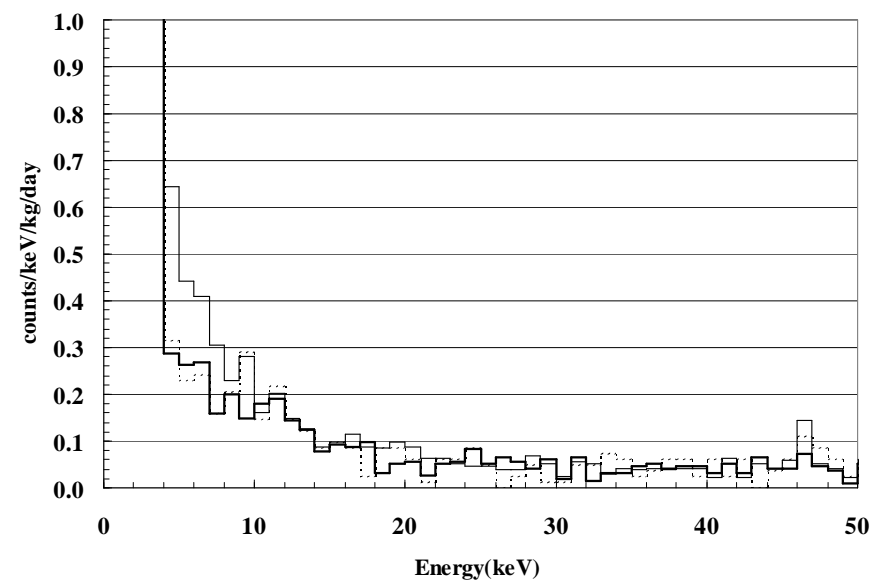

Figure 1. Normalized low energy spectrum of the IGEX RG-II detector corresponding to the three different neutron shielding conditions mentioned in the text: $236 \mathrm{~kg}$ d with $20 \mathrm{cms}$ of polyethylene (thin solid line), $194 \mathrm{~kg} \mathrm{~d}$ with $40 \mathrm{cms}$ of polyethylene and borated water (thick solid line) and $82 \mathrm{~kg}$ day with $80 \mathrm{cms}$ of polyethylene and borated water (dashed line).

in the next section. For more details on the IGEX shielding we refer to ${ }^{5}$ and ${ }^{6}$.

In addition to the data acquisition system used in the first runs (described in ${ }^{5}$ ), a specific pulse shape analysis was implemented for subsequents sets of data ${ }^{6}$. The charge pulse shapes of each event before and after amplification are recorded by two $800 \mathrm{MHz}$ LeCroy 9362 digital scopes. These are analyzed one by one by means of a method based on wavelet techniques $^{7}$ which allows us to get the probability of this pulse to have been produced by a random fluctuation of the baseline. This probability is used as a criterium to reject events coming from electronic noise or microphonics. According to the calibration of the method, it works very efficiently for events above $4 \mathrm{keV}$.

\section{Status}

As has been stressed in previous papers ${ }^{5}$, the sensitivity of the experiment can be substantially improved if modest reduction of background at low energies is achieved. Currently the main concern of the collaboration is the identification of the low energy background in order to design strategies that allow us to reduce it. Several studies based on simulation and experimentation are still under work, but some improvements in that sense have 


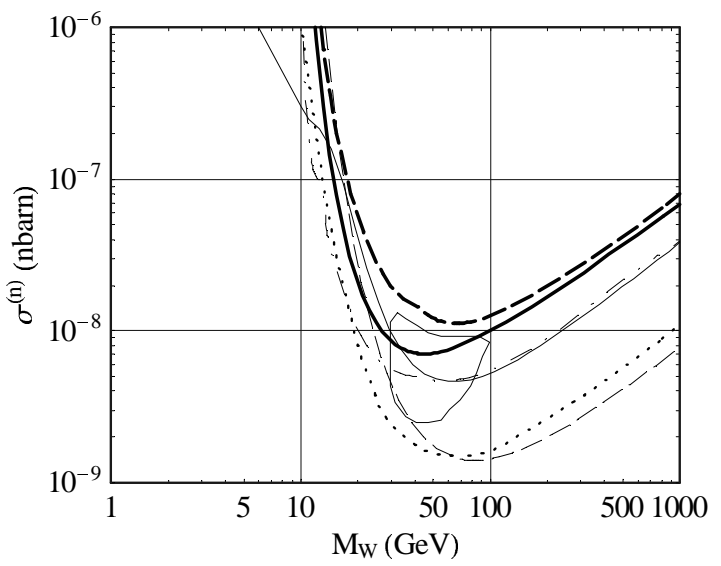

Figure 2. IGEX-DM exclusion plot for spin-independent interaction obtained with the data before (thick dashed line) and after (thick solid line) the neutron shielding improvement, compared with the results from other experiments which exclusions also enter in the DAMA region: $\mathrm{CDMS}^{9}$ (thin dot-dashed line), EDELWEISS ${ }^{10}$ (thin dashed line). Also included is the very recent curve presented by the ZEPLIN collaboration in this same conference ${ }^{12}$ (dotted line). The thin solid line is the exclusion line obtained by DAMA NaI-0 by using Pulse Shape Discrimination ${ }^{11}$. The closed line corresponds to the $(3 \sigma)$ annual modulation effect reported by the DAMA collaboration (including NaI- $1,2,3,4$ runnings) ${ }^{2}$.

been already achieved and recently published ${ }^{6}$. In Fig. 1 we shown the spectra taken before (thin line) and after (thick line) the first improvement of the neutron shielding. Those spectra are the same as the ones published in ${ }^{5}$ and ${ }^{6}$ respectively, but with more statistics added. The simulations that motivated this improvement are in qualitative agreement with the reduction of the experimental background actually obtained (that amounts to a $50 \%$ in the first energy bins) although the remaining low energy population of events is not yet fully identified. More recently data has been taken with an even thicker neutron moderator wall $(80 \mathrm{cms})$, obtaining a similar level of background, as plotted in Fig. 1 (dotted line). The fact that this second change does not improve the background is also consistent with our simulations and with the fact the neutrons from the rock have been practically shielded out from the IGEX background. On the other hand, simulations taking into account surrounding contaminations of typical radio-isotopes $\left({ }^{60} \mathrm{Co},{ }^{40} \mathrm{~K},{ }^{238} \mathrm{U} /{ }^{222} \mathrm{Rn},{ }^{232} \mathrm{Th},{ }^{210} \mathrm{~Pb}, \ldots\right)$ only explain a flat background of $\sim 0.04 \mathrm{c} / \mathrm{keV} / \mathrm{kg} / \mathrm{d}$ as the one experimentally obtained above $\sim 20 \mathrm{keV}$. The identification of the remaining events that populate the low energy region above this level (accounting for up to $\sim 0.2 \mathrm{c} / \mathrm{kev} / \mathrm{kg} / \mathrm{d}$ between 4 and 10 


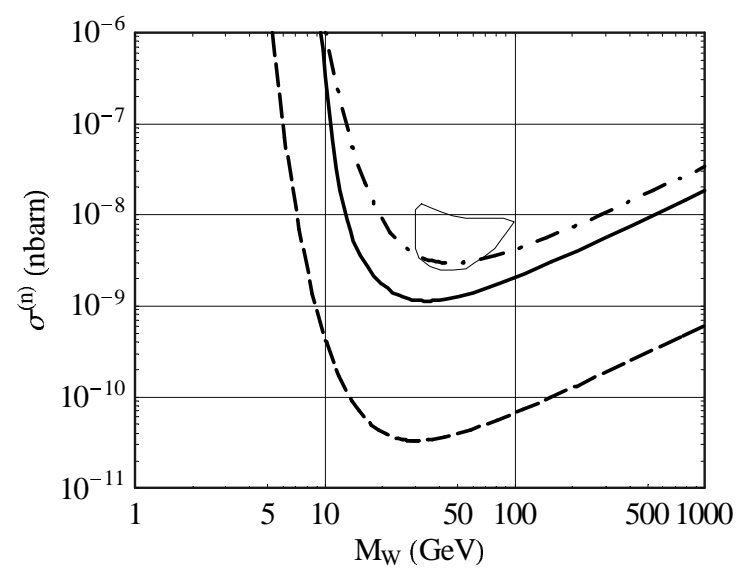

Figure 3. IGEX-DM projections are shown for a flat background rate of $0.1 \mathrm{c} / \mathrm{keV} / \mathrm{kg} /$ day (dot-dashed line) and $0.04 \mathrm{c} / \mathrm{keV} / \mathrm{kg} /$ day (solid line) down to the threshold at $4 \mathrm{keV}$, for $1 \mathrm{~kg}$ year of exposure. The exclusion contour expected for GEDEON is also shown (dashed line) as explained in the text.

$\mathrm{keV}$ ) is currently the main goal of the experiment. One possibility under consideration for the origin of these events is an internal contamination of tritium. On the other hand, the effect of muon-induced neutrons is being studied in detail, the preliminary conclusion being that they can hardly explain this population. Let us say that all these studies will allow also to set the prospects of the projected extension of IGEX for dark matter searches, the GEDEON project.

The exclusion plots are derived from the both recorded spectra (before and after the first modification) following the same set of hypothesis and parameters used in previous papers $\left(\right.$ see $^{5}$ ) and are shown in Fig. 2 (thick solid and dashed lines). They are compared with other recent exclusions.

\section{Prospects}

As stated before, background identification works are still in progress and some strategies are being considered to further reduce the low energy background. If this reduction is achieved, very interesting perspectives can be set for IGEX. In Fig. 3 we plot the exclusions obtained with a flat background of $0.1 \mathrm{c} / \mathrm{kg} / \mathrm{keV} /$ day (dot-dashed line) and of $0.04 \mathrm{c} / \mathrm{kg} / \mathrm{keV} /$ day (solid line) down to the current $4 \mathrm{keV}$ threshold for an exposure of $1 \mathrm{~kg}$ year. In particular, the complete DAMA region could be tested with a moderate improvement of the IGEX performances. The dashed line in Fig. 3 
corresponds to a flat background of $0.002 \mathrm{c} / \mathrm{kg} / \mathrm{keV} /$ day down to a threshold of $4 \mathrm{keV}$ and $24 \mathrm{~kg}$ y of exposure, which are the parameters expected for GEDEON (GErmanium DEtectors in ONe cryostat), a new experimental project on WIMP detection using larger masses of natural germanium planned as an extension of the IGEX dark matter search (see ref. ${ }^{6}$ ). GEDEON would be massive enough ${ }^{13}$ to search also for the WIMP annual modulation effect and explore positively an important part of the WIMP parameter space including the DAMA region.

\section{Conclusions}

The IGEX dark matter experiment is carrying a thorough study of its low energy experimental background which after the last improvements of the neutron shielding is already at the level of $\sim 0.2 \mathrm{c} / \mathrm{keV} / \mathrm{kg} /$ day (between 4 and $10 \mathrm{keV}$ ), the lowest low energy raw background achieved up to now. The possibility of further background rejection sets interesting prospects for the experiment. The GEDEON project, conceived as an natural extension of the IGEX-DM experiment will have a substantially improved background and will be able to explore the annual modulation signature.

\section{References}

1. See for instance A. Morales, Nucl. Phys. Proc. Suppl. 110 (2002) 39 [arXiv:astro$\mathrm{ph} / 0112550]$.

2. R. Bernabei et al. [DAMA Collaboration], Phys. Lett. B 450 (1999) 448; R. Bernabei et al. [DAMA Collaboration], Phys. Lett. B 480 (2000) 23.

3. C. E. Aalseth et al. [IGEX Collaboration], Phys. Rev. C 59 (1999) 2108.

4. D. Gonzalez et al. [IGEX Collaboration], Nucl. Phys. Proc. Suppl. 87 (2000) 278.

5. A. Morales et al. [IGEX Collaboration], Phys. Lett. B 489 (2000) 268 [hepex/0002053].

6. A. Morales et al., Phys. Lett. B 532 (2002) 8 [arXiv:hep-ex/0110061].

7. Paper in preparation. A brief description can be found in ${ }^{6}$.

8. L. Baudis et al., Phys. Rev. D 59 (1999) 022001 [hep-ex/9811045].

9. R. Abusaidi et al. [CDMS Collaboration], Phys. Rev. Lett. 84 (2000) 5699 [astro$\mathrm{ph} / 0002471]$.

10. A. Benoit et al. [EDELWEISS Collaboration], Phys. Lett. B 513 (2001) 15 [astro$\mathrm{ph} / 0106094]$.

11. R. Bernabei et al., Phys. Lett. B 389 (1996) 757.

12. Talk given at IDM2002, these proceedings.

13. S. Cebrian et al., Astropart. Phys. 14 (2001) 339 [hep-ph/9912394]. 